\title{
The influence of insulation of walls of industrial objects on thermal regime at the heating system of gas infrared radiators
}

\author{
Tatiana Nagornova ${ }^{1, *}$, and Vladimir Emelenchuk ${ }^{1}$ \\ ${ }^{1}$ National Research Tomsk Polytechnic University, 634050 Tomsk, Russia
}

\begin{abstract}
The results of a numerical study of the process of heat transfer from the gas infrared emitters in the heated accommodation are represented. Simulation was conducted taking into account the heat withdrawal in the enclosing constructions and of heat exchange with the environment. The estimation of the average values of temperatures of air indoors in the dependence on the different intensity of heat withdrawal into the vertical walls is carried out (when the layer of insulation is present, and without it).
\end{abstract}

\section{Introduction}

The contemporary tendencies of development of science and technology are directed toward strengthening of the economic characteristics of resource use [1,2]. The savings of the energy and thermal resources has great significance [3-6]. The minimization of expenditures for the heating of large production accommodations will make it possible to reduce capital investments and prime cost of production. At present, widely adapt alternative water of heating system, for example, gas infrared emitters (GIE) [7]. Besides the replacement of heating system possible to use different design methods of an improvement in the thermal insulation properties of premises - the internal isolation of the lateral enclosing constructions. The joint application of GIE and vertical enclosing constructions, covered with thermal insulation materials, is urgent task. Reduction in the intensity of heat withdrawal through the walls of accommodation can lead to substantial changes in the thermal condition. Similar studies earlier were not conducted, but they are sufficiently interesting. The purpose of the work - quantitative assessment is the influence of the heat insulation of walls on the thermal condition of premises with the heating system on the base of gas infrared emitters.

\section{Formulation of the problem}

To describe the conjugate heat transfer in a room of an industrial facility heated by GIE, a rectangular region (fig. 1) with a source of radiant heating was chosen. As a basic system of dimensionless differential equations describing the process of natural convection, a mathematical model similar to [8] is adopted. The equations of thermal conductivity for

\footnotetext{
* Corresponding author: tania@tpu.ru
} 
each of the elements of the enclosing constructions were solved. For a gas, the differential Navier-Stokes and energy equations were considered (in the transformed variables "the function of current - vorticity - temperature"). On the external contour of the solution region, boundary conditions were adopted, taking into account heat exchange with the surrounding medium. The boundary conditions of the first kind were set on the surface of the radiant-heating source. At the lower boundary of the medium (the lower concrete base), the heat flux coming from the GIE was taken into account.

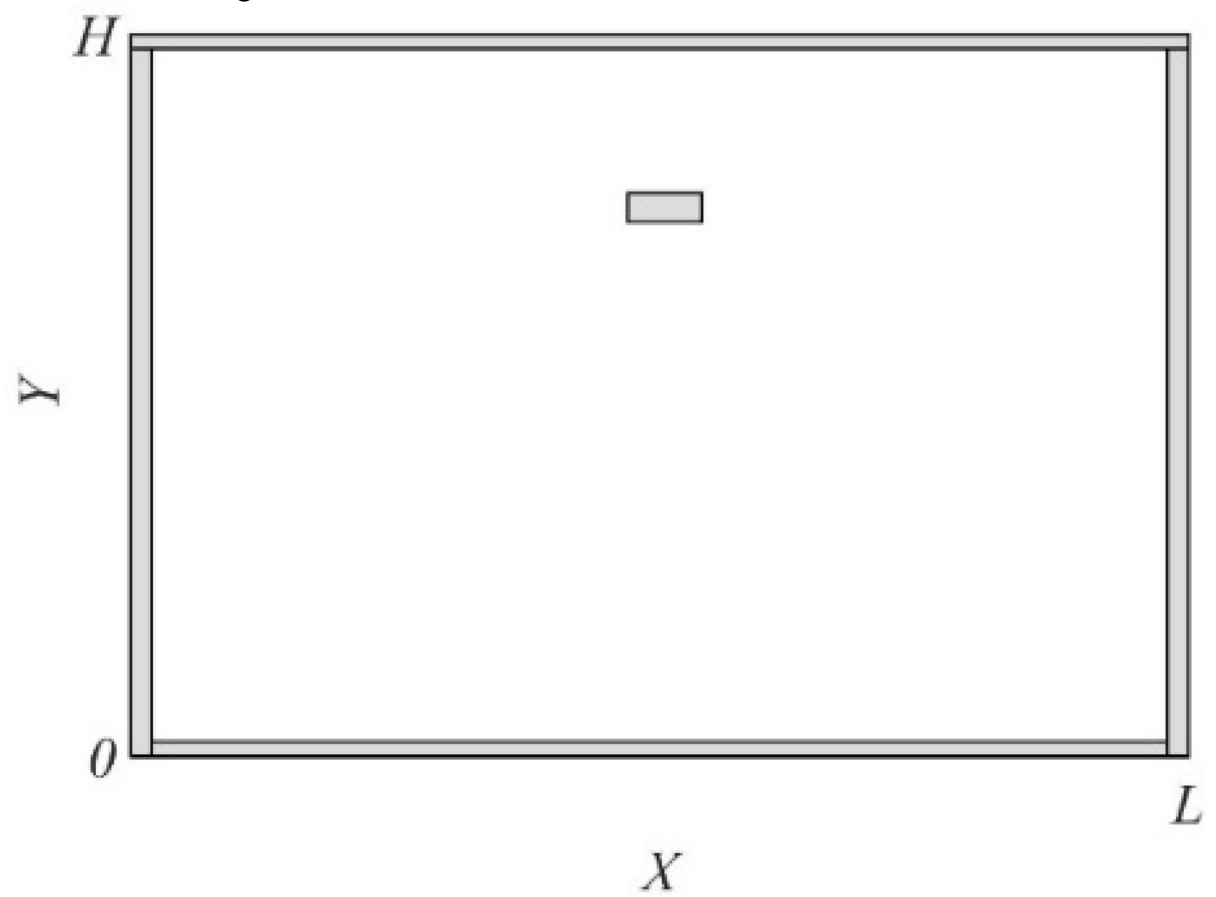

Fig. 1. Area of solution of the problem: $L, H$ - transverse size and height of the solution region, respectively.

Equations with the appropriate initial and boundary conditions were solved by the finite-difference method on the uniform grid. To solve the system of difference equations, the sweep method and the variable direction scheme are used [9]. Was used algorithm $[8,10]$, developed for the numerical solution of the problems of natural convection in closed rectangular regions with local energy sources.

\section{Results and discussion}

In Fig. 2.3 shows the dynamics of the change in temperature fields and streamlines at different time values for two different heat dissipation options in enclosing structures. The conditions for heat insulation on the vertical walls and its absence were examined. 

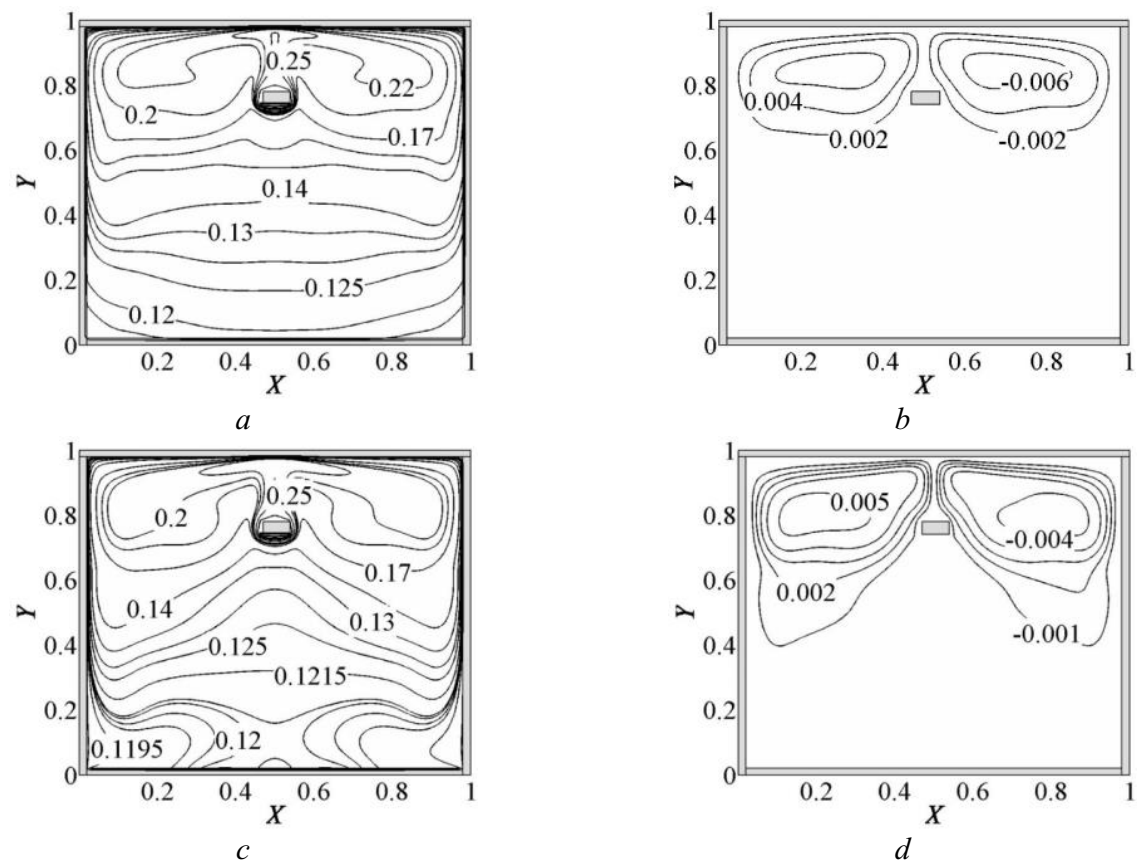

Fig. 2. Temperature fields and isolines of the current function at $\tau=120 \cdot 10^{3}$ in the case of insulation $(a, b)$ of vertical enclosing structures and without it $(c, d)$.
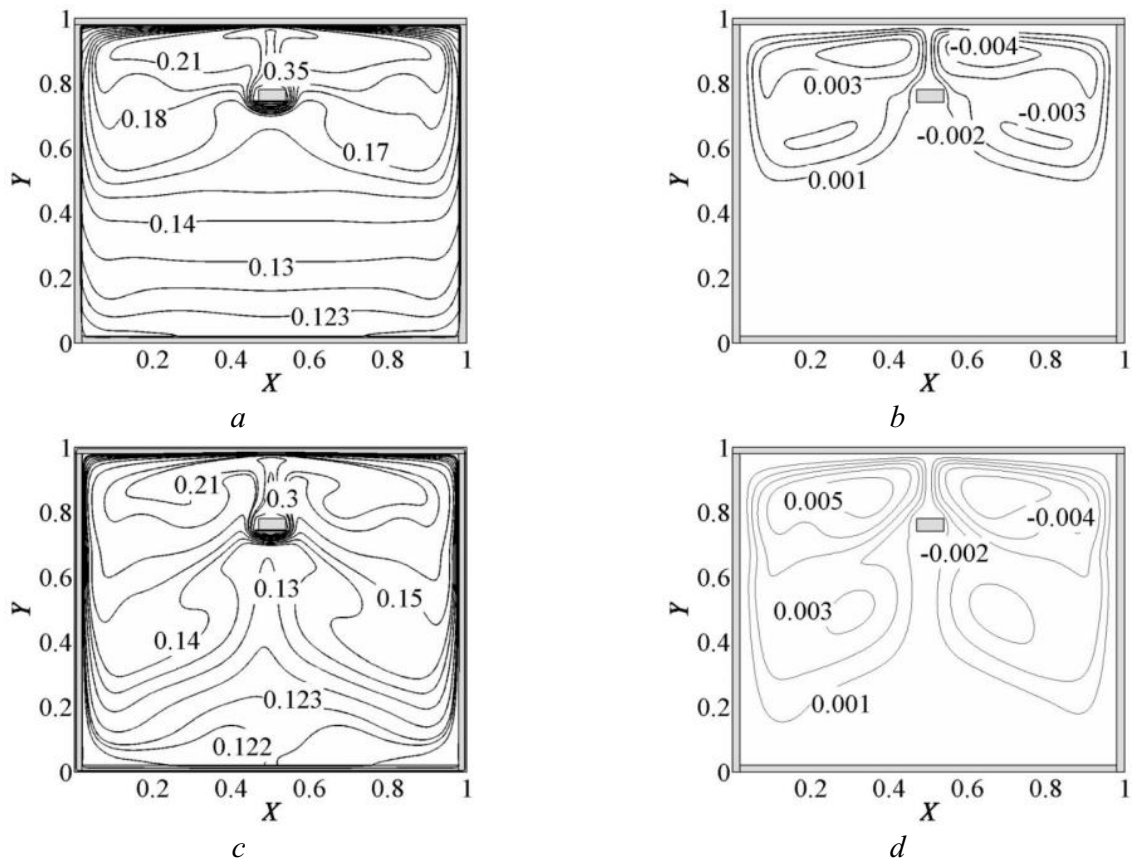

Fig. 3. Temperature fields and isolines of the current function at $\tau=240 \cdot 10^{3}$ in the case of insulation $(a, b)$ of vertical enclosing structures and without it $(c, d)$.

Analysis of the results Fig. 2 and 3 it shows that the introduction of changes in the construction of the enclosing constructions has an essential effect on the structure of 
convective flows in heated of premises and the temperature fields (Fig. 4). In the case of wall insulation, there is no formation of vortex structures under the source of radiant heating and, accordingly, there is no intensive mixing of air masses. The stratification of isotherms near the lower "air-floor" interface has been established (Figs. 2, 3).

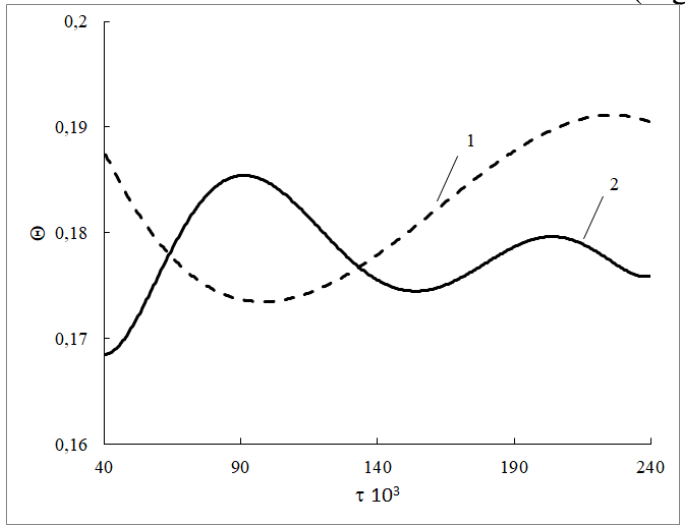

Fig. 4. Dependence of the average temperature of the air environment on the time when the GIE is operating with the use of insulation (1) and in the case of its absence (2).

The results of the numerical simulation (Figure 4) also show a variety of changes in the mean temperatures over time. In the time period $70 \cdot 10^{3}$ and $130 \cdot 10^{3}$, the values of $\Theta$ for the two variants coincide. Nonmonotonic changes in mean temperatures with time are caused by the processes of heat withdrawal to the enclosing constructions. In the initial period of time, the structure of the flow of heated gas flows upwards. But with an increase $\tau$ in the value of mean temperatures in the conditions for the heat insulation of vertical boundaries it becomes more, increases difference numerical values $\Theta$ for two versions in question.

\section{Conclusion}

The results of theoretical studies of heat transfer processes in a building heated by a gas infrared emitter allow us to say that insulating the vertical enclosing structures leads to a significant change in the structure of the flows and temperature distributions of heated air in the room. In this case, in the course of time the influence of heat withdrawal becomes ever more essential. The change in the structure of the flow in time leads to an uneven increase in the average air temperature.

The work was supported by the Russian President's grant (Scientific School project 7538.2016.8).

\section{References}

1. Federal Law from 23.11, N 261-FZ (Red. 03.07.2016) "On Energy Saving and Improvement of Energy Efficiency" (2009) [in russian]

2. European Parliament and the Council of European Parliament, Directive 2010/31/EU of the Parliament and of the Council of 19 May 2010 on the energy performance of building (recast), Off. J. Eur. Union (2010) 
3. Xiaojing Meng, Yi Wang, Tiening Liu, Xiao Xing, Yingxue Cao, Jiangping Zhao, Appl. Therm. Eng., 96, 473-480 (2016), DOI: 10.1016/j.applthermaleng.2015.11.105

4. P. Rohdin, B. Moshfegh, Build. Environ., 46, 2365-2374 (2011), DOI: 10.1016/j.buildenv.2011.05.019

5. X. Ouyang, B. Lin, Energy and Build., 109, 316-327 (2015), DOI: 10.1016/j.enbuild.2015.09.068

6. E. Rebollo, C.A. Platero, F.R. Blanquez, F. Blazquez, J.A. Gaonab, Procedia Comput. Sci., 83, 886-893 (2016), DOI: 10.1016/j.procs.2016.04.180

7. K.-N. Rhee, B.W. Olesen, K.W. Kim, Build. Environ., 112, 367-381 (2017), DOI: 10.1016/j.buildenv.2016.11.030

8. G.V. Kuznetsov, N.I. Kurilenko, V.I. Maksimov, G.Ya. Mamontov, T.A. Nagornova, J. Eng. Phys. Thermophys., 86, 3 (2013), DOI: 10.1007/s10891-013-0863-6

9. T. Cebeci, Analysis of Turbulent Flows with Computer Programs (The Boulevard, Langford Lane, Kidlington, Oxford, 2013)

10. M.A. Sheremet, Thermophys. Aeromechanics, 18, 1 (2011), DOI: 10.1134/S0869864311010124 